\title{
Low-Frequency Stability of the Razor Blade Tested MFIE
}

\author{
I. Bogaert*, F. P. Andriulli ${ }^{\dagger}$ and K. Cools ${ }^{\ddagger}$
}

\begin{abstract}
Razor blade testing schemes have been proposed in the past for both the EFIE and MFIE. The regularity of these testing functions is, strictly speaking, not sufficient for the discretization to be conforming. However, as will be shown in the contribution, it does yield physical solution currents at low frequencies. This is similar to the low-frequency behavior of the mixed discretization of the MFIE. Nevertheless, in this testing scheme, there is no refined mesh, the impedance integrals are triple integrals instead of quadruple integrals and, in addition, the testing functions are constant instead of linear on their support.
\end{abstract}

\section{Introduction}

For a long time, the widespread use of the magnetic field integral equation (MFIE) has been hindered by its apparently inferior accuracy [1]. However, in recent years, it has been shown that this accuracy problem has more to do with the testing scheme that is predominantly used than with the properties of the MFIE itself. Indeed, it was shown that a mixed discretization scheme, in which the MFIE is tested using rotated Buffa-Christiansen (BC, [2]) or Chen-Wilton (CW, [3]) functions, delivers a much better accuracy $[4,5]$ than the standard discretization scheme. In addition, the mixed discretization yields physical solutions at low frequencies $[6,7]$, which is in stark contrast to the standard scheme. A drawback of the mixed scheme is the need for a barycentrically refined mesh, which increases the number of triangle-triangle interactions that needs to be evaluated for each impedance matrix element. In this contribution, an alternative testing scheme, which was explained for example in [8], is shown to yield physical solution currents at low frequencies (LF) as well.

The effectiveness of the testing scheme is related to the fact that the Rao-Wilton-Glisson (RWG, [9]) basis functions are actually smoother than what is required for conformity. Indeed, when $\Gamma$ is the boundary of a scatterer, the RWGs are elements of $H_{\mathrm{div}}^{0}(\Gamma)$ (and even some higher-index Sobolev

\footnotetext{
*Department of Information Technology, Ghent University, Sint-Pietersnieuwstraat 41, 9000 Ghent, Belgium, email:Ignace. Bogaert@intec.UGent . be

${ }^{\dagger}$ Microwave Department of Telecom Bretagne, Institut Mines-Telecom, Brest, France

$\ddagger$ Electrical Systems and Optics Research Division, University of Nottingham, Nottingham, U.K.
}

spaces), while the requirement for conformity is being an element of $H_{\mathrm{div}}^{-\frac{1}{2}}$. If the boundary is smooth, it makes sense that some of this regularity can be be used to ease the regularity requirements of the testing function. The razor blade testing finite element space is not sufficiently regular to be in $H_{\text {div }}^{-\frac{1}{2}}$, but nevertheless retains many of the desirable characteristics of the BCs or CWs. For example, the number of loops in the finite element space is equal to the number present in the $\mathrm{BC}$ or $\mathrm{CW}$ spaces.

\section{Razor Blade Testing Scheme}

We first define a current distribution on the line between the points $\boldsymbol{r}_{1}$ and $\boldsymbol{r}_{2}$ :

$$
\boldsymbol{I}=\frac{\boldsymbol{r}_{2}-\boldsymbol{r}_{1}}{\left\|\boldsymbol{r}_{2}-\boldsymbol{r}_{1}\right\|}, \forall \boldsymbol{r}=t \boldsymbol{r}_{1}+(1-t) \boldsymbol{r}_{2}, t \in[0,1]
$$

For all other points, the current is zero. As such, the function represents an infinitely thin wire carrying a unit current between the points $\boldsymbol{r}_{1}$ and $\boldsymbol{r}_{2}$. It is clear that for every edge on the mesh, such wire current can be constructed by using the begin and end points of the edge as $\boldsymbol{r}_{1}$ and $\boldsymbol{r}_{2}$. These current distributions will be called $\boldsymbol{I}_{n}, \forall n \in[1, E]$, with $E$ the number of edges. The current $\boldsymbol{I}$ can also be written in a distributional form in $\mathbb{R}_{3}$. For example, when $\boldsymbol{r}_{1}=[0,0,0]$ and $\boldsymbol{r}_{2}=[1,0,0]$, the following distribution is found:

$$
\boldsymbol{I}(\boldsymbol{r})=H(x) H(1-x) \delta(y) \delta(z) \hat{\boldsymbol{u}}_{x} .
$$

Let us now move on to the exterior MFIE (the interior MFIE can be handled in the same fashion), which is given by

$$
\hat{\boldsymbol{n}} \times\left[\boldsymbol{h}^{i}(\boldsymbol{r})+\lim _{\boldsymbol{r} \rightarrow \Gamma} \int_{\Gamma} \nabla G_{k}\left(\boldsymbol{r}-\boldsymbol{r}^{\prime}\right) \times \boldsymbol{j}\left(\boldsymbol{r}^{\prime}\right) \mathrm{d} S^{\prime}\right]=0 .
$$

Here, the limit of the observation point $\boldsymbol{r}$ to the boundary $\Gamma$ is taken from the inside of the scatterer towards the surface. $\hat{\boldsymbol{n}}$ is the outward-pointing normal unit vector. The current $\boldsymbol{j}(\boldsymbol{r})$ will be expanded in RWGs

$$
\boldsymbol{j}(\boldsymbol{r})=\sum_{m=1}^{E}[\boldsymbol{J}]_{m} \boldsymbol{W}_{m}(\boldsymbol{r})
$$


that are defined without the edge length factor:

$$
\boldsymbol{W}_{m}(\boldsymbol{r})= \pm \frac{\boldsymbol{r}-\boldsymbol{r}_{ \pm}}{2 A_{ \pm}}, \forall \boldsymbol{r} \in \Delta_{ \pm}
$$

Here, the $\Delta_{ \pm}$are the two triangles (with surface area $A_{ \pm}$) on which the RWG is defined.

The razor blade testing scheme now amounts to testing equation (3) with $\hat{\boldsymbol{n}} \times \boldsymbol{I}_{n}$. It should be noted that the surface normal appearing in this formula is not well-defined because the support of $\boldsymbol{I}_{n}$ is exactly on the edge. Indeed, the normal of either triangle can be used, yielding a differently oriented testing function. This ambiguity can be resolved, however, by canceling the cross product with the one appearing in the MFIE. When this is done, the magnetic field itself is tested with the wire currents $\boldsymbol{I}_{n}$, which is well-defined. Finally, this yields the matrix equation

$$
\mathrm{P} \cdot \boldsymbol{J}=\boldsymbol{H},
$$

with

$[\mathrm{P}]_{n, m}=\lim _{\epsilon \rightarrow 0} \int_{\Gamma} \boldsymbol{I}_{n}(\boldsymbol{r}+\epsilon \hat{\boldsymbol{n}}) \cdot \int_{\Gamma} \nabla G_{k}\left(\boldsymbol{r}-\boldsymbol{r}^{\prime}\right) \times \boldsymbol{R}_{m}\left(\boldsymbol{r}^{\prime}\right) \mathrm{d} S^{\prime}$.

and

$$
[\boldsymbol{H}]_{n}=-\int_{\Gamma} \boldsymbol{I}_{n}(\boldsymbol{r}) \boldsymbol{h}^{i}(\boldsymbol{r}) \mathrm{d} S .
$$

It is worthwhile to point out that the integral appearing in (7) is three-dimensional, not four dimensional, which is nice from a computational point of view.

\section{Impedance Integrals}

While several historical implementations of the razor blade testing scheme for the MFIE have been using point matching, i.e. a one-point integration rule for the testing, this is not a suitable technique for obtaining the low-frequency behavior analyzed in this work. Instead, the full three dimensional integration must be carried out. This integration is not particularly demanding from a computational perspective, however, because the hardest integral, i.e. the self-patch, can be computed analytically. Indeed, following from results in [8],

- If a wire current is located on one of the four outer edges of an RWG support, then the associated impedance integral only contains a contribution from the triangle that is not coplanar to the wire. Indeed, the triangle that shares an edge with the wire current is coplanar and, in addition, there is no normal component of the current. Hence, this contribution is zero.
- The self-patch, i.e. the impedance integral between a wire current and the RWG defined on the two adjacent triangles, can be evaluated analytically as

$$
[\mathrm{P}]_{n, n}=1-\frac{\Omega_{n}}{2 \pi} .
$$

Here, $\Omega_{n}$ is the angle between the two triangles, measured through the interior of the scatterer. A graphical representation of this angle is shown in Figure 1.

Summarizing, the diagonal elements of the impedance matrix can be computed analytically, even for the dynamic Green's function. Finally, it is also worthwhile to point out that, using the analytical techniques presented in [10], it is possible to reduce the dimensionality of the remaining impedance integrals to two.

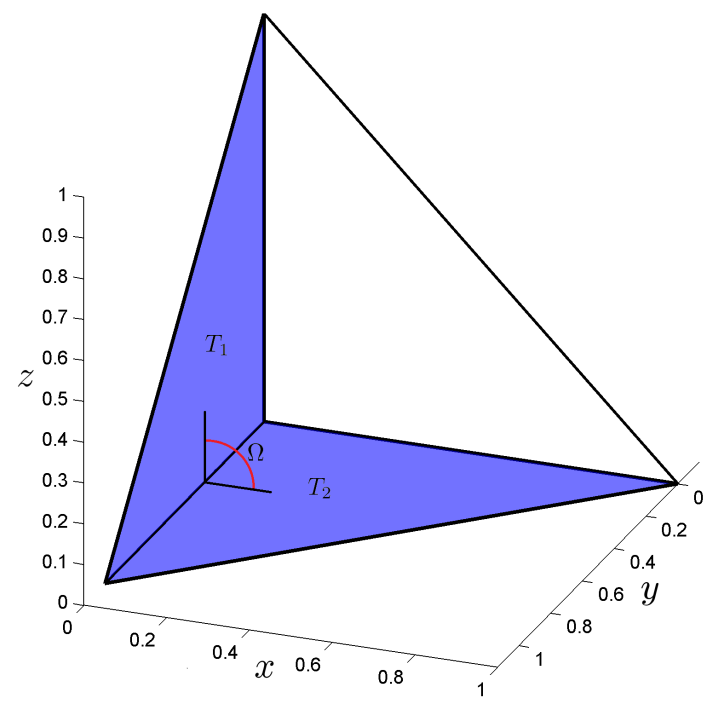

Figure 1: The angle $\Omega$ between triangles $T_{1}$ and $T_{2}$, as measured through the interior of a tetrahedral scatterer.

\section{Low-Frequency Stability}

The low-frequency stability of the razor blade testing scheme will now be investigated. In the light of [6], for a simply connected scatterer, it is necessary and sufficient to prove that the razor blade finite element space contains $V-1$ stars and $F-1$ loops. Here, $V$ and $F$ are the number of vertices and faces in the mesh respectively. To check this, it is necessary to count the number of degrees of freedom in the charge space associated with the razor blade testing functions. As is easily seen, the divergence of a razor blade is zero everywhere, except for two Dirac delta distributions (with opposite sign) 
at the two vertices that are connected by the razor blade. Using charge neutrality, this allows us to conclude that there are $V-1$ linearly independent charges. As a consequence, there are $F-1$ loops, hence the requirement for low-frequency stability is met for simply connected structures. In the same spirit as [7], it can also be concluded that this testing scheme for the MFIE on non-simply connected scatterers will lead to an exact null-space in the DC limit, which is physical because it is also found in the continuous MFIE [11].

\section{$5 \quad$ Numerical Results}

The razor blade testing method has been implemented in $\mathrm{C}++$. It is clear that the implementation of this testing scheme is significantly less complex than the one with $\mathrm{BC}$ or $\mathrm{CW}$ functions due to the absence of the barycentrically refined mesh. In addition, the impedance integrals are triple integrals, as opposed to the usual quadruple integrals. Finally, the test functions are constant on their support, as opposed to linear for the $\mathrm{BC}$ or $\mathrm{CW}$ functions.

To test the low-frequency behavior for the simply connected case, a spherical scatterer with radius $1 \mathrm{~m}$ was illuminated by a $y$-polarized plane wave propagating in the $x$ direction. The wave number was varied between $10^{-5} \mathrm{~m}^{-1}$ and $1 \mathrm{~m}^{-1}$. The $L_{2}$ norm of the loop-loop block of the impedance matrix $P$ (see block $P^{l l}$ in equation (9) from [6]) was computed for each wave number, as well as the $L_{2}$ norm of the nonsolenoidal part of the solution current. Both results are plotted as a function of the wave number in Figure 2 and behave as expected, i.e. the loop-loop block of the impedance matrix is proportional to $\omega^{2}$ and the nonsolenoidal part of the solution current is proportional to $\omega$. This is physical at low frequencies because the charge density associated with this nonsolenoidal part is constant (as a function of $\omega$ ) in this case, which is consistent with the plane wave incoming field.

To test the existence of a null-space at DC for the non-simply connected case, a similar wave number sweep was made for a toroidal scatterer, discretized using 459 RWGs and displayed in figure 5. For each wave number, the smallest singular value was plotted in Figure 3. Again, the result is as expected from [7].

\section{Conclusion}

The razor blade testing scheme for the MFIE was analyzed in the context of low-frequency scattering. The razor blade testing does not require a barycentrically refined mesh but retains the desirable low-

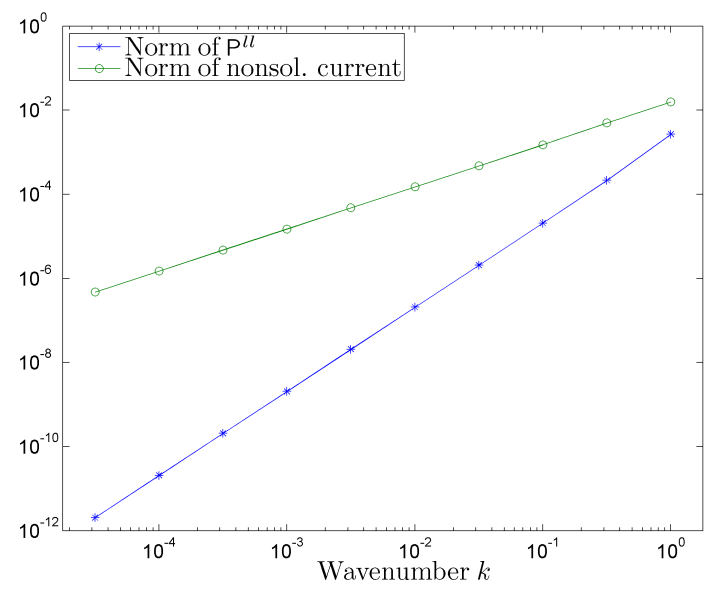

Figure 2: The frequency scaling of the loop-loop block of the matrix $\mathrm{P}$ and of the star part of the solution current.

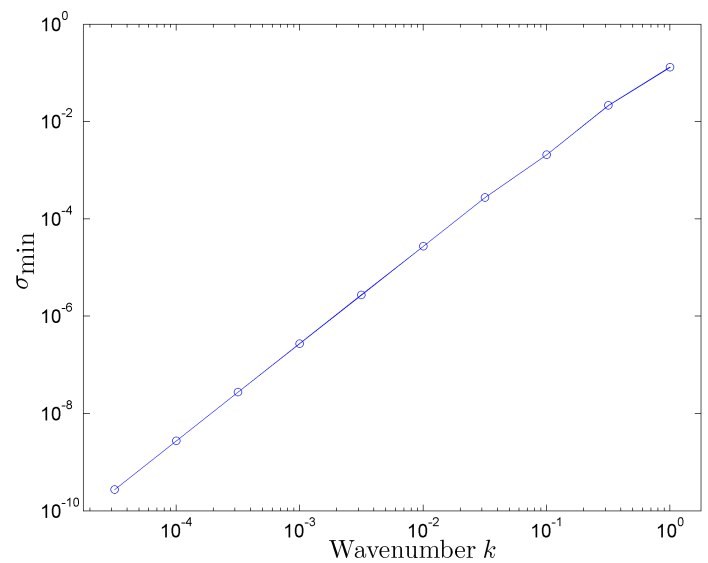

Figure 3: The frequency scaling of the smallest singular value of the impedance matrix for the toroidal scatterer shown in Figure 5.

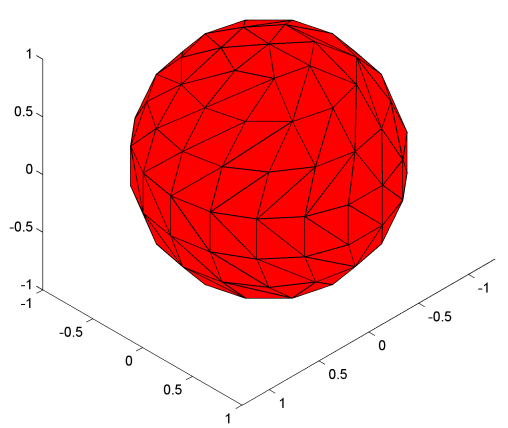

Figure 4: The sphere mesh used for generating the results in Figure 2. The radius is $1 \mathrm{~m}$ and the number of edges is 318 . 


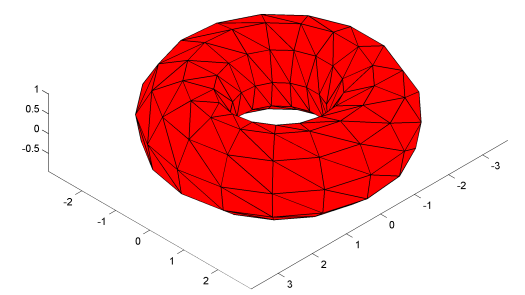

Figure 5: The toroidal mesh used for generating the results in Figure 3. The number of edges is 459 .

frequency characteristics of the mixed discretization scheme based on $\mathrm{BC}$ or $\mathrm{CW}$ functions. An additional computational advantage is that the selfpatch can be computed analytically without effort. In addition, the simple form of the testing functions allows for an easy implementation of the other impedance integrals. Numerical results have shown that the theoretically expected low-frequency behavior is realized in practice.

\section{Acknowledgments}

The work of Ignace Bogaert was supported by a postdoctoral grant from the Fund for Scientific Research Flanders (FWO-Vlaanderen).

\section{References}

[1] Y. Zhang, C. T.J., W. Chew, and J. Zhao, "Magnetic field integral equation at very low frequencies," IEEE Transactions on Antennas and Propagation, vol. 51, no. 8, pp. 1864-1871, Aug 2003.

[2] A. Buffa and S. H. Christiansen, "A dual finite element complex on the barycentric refinement," Math. of Comp., vol. 260, pp. 17431769, 2007.

[3] Q. Chen and D. Wilton, "Electromagnetic scattering by threedimensional arbitrary complex material/conducting bodies," Proceedings of the IEEE Symposium on Antennas and Propagation, vol. 2, pp. 590-593, 1990.

[4] K. Cools, F. Andriulli, F. Olyslager, and E. Michielssen, "Improving the MFIE's accuracy by using a mixed discretization," in $A n$ tennas and Propagation Society International Symposium, June 2009, pp. 1-4.

[5] K. Cools, F. Andriulli, D. De Zutter, and E. Michielssen, "Accurate and Conforming Mixed Discretization of the MFIE," IEEE Antennas and Wireless Propagation Letters, vol. 10, pp. 528-531, 2011.
[6] I. Bogaert, K. Cools, F. P. Andriulli, J. Peeters, and D. De Zutter, "Low Frequency Stability of the Mixed Discretization of the MFIE," in Proceedings of the 5th European Conference on Antennas and Propagation, Rome, Italy, 11-15 April 2011.

[7] I. Bogaert, K. Cools, F. Andriulli, and D. De Zutter, "Low Frequency Scaling of the Mixed MFIE for Scatterers with a Non-Simply Connected Surface," in Proceedings of the International Conference on Electromagnetics in Advanced Applications, Torino, Italy, 12-17 September 2011.

[8] A. Peterson, S. Ray, and R. Mittra, Computational methods for electromagnetics. New York: IEEE, 1998.

[9] S. D. Rao, D. R. Wilton, and A. W. Glisson, "Electromagnetic scattering by surfaces of arbitrary shape," IEEE Trans. Antennas Propag., vol. AP-30, no. 3, May 1982.

[10] I. Bogaert, "On the Analytical Treatment of Singular Impedance Integrals in Electromagnetics," in Proceedings of the International Conference on Electromagnetics in Advanced Applications, Turin, Italy, 9-13 September 2013.

[11] K. Cools, F. Andriulli, F. Olyslager, and E. Michielssen, "Nullspaces of mfie and calderón preconditioned efie operators applied to toroidal surfaces," Antennas and Propagation, IEEE Transactions on, vol. 57, no. 10, pp. 3205-3215, 2009. 